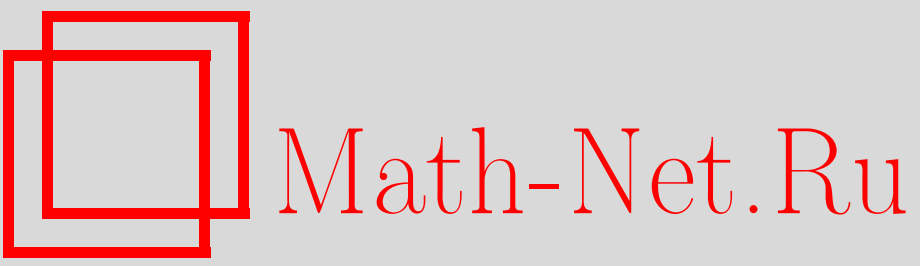

И. А. Дынников, С. В. Смирнов, Точно решаемые циклические $q$-цепочки Дарбу, УМH, 2002, том 57, выпуск 6, 183-184

DOI: https://doi.org/10.4213/rm583

Использование Общероссийского математического портала Math-Net.Ru подразумевает, что вы прочитали и согласны с пользовательским соглашением

http://www.mathnet.ru/rus/agreement

Параметры загрузки:

IP: 54.198 .187 .58

26 апреля 2023 г., 16:48:05 


\title{
ТОЧНО РЕШАЕМЫЕ ЦИКЛИЧЕСКИЕ $q$-ЦЕПОЧКИ ДАРБУ
}

\author{
И. А. Дынников, С. В. Смирнов
}

Цепочкой Дарбу назьвается последовательнсть самосопряженных дифференциалных операторов $L_{1}, L_{2}, \ldots$ на прямой, связанных преобразованиями Дарбу:

$$
L_{j}=A_{j} A_{j}^{+}-\alpha_{j}=A_{j-1}^{+} A_{j-1},
$$

где $A_{j}=-d / d x+f_{j}(x)$ - дифференциалшные операторы первого порядка. Цепочка называется циклической периода (или длины $r$ ), если $L_{j+r}=L_{j}, j=1,2, \ldots$ В частном случае $r=1$ оператор $L+\frac{\alpha}{2}$ является гармоническим осциллятором.

Циклические цепочки Дарбу приводят к интегрируемьм системам дифференциальных уравнений на функции $f_{j}$, которые изучались в [1]. Принципиально отличаются случаи $\alpha=0$ и $\alpha \neq 0$, где $\alpha=\sum_{j=1}^{r} \alpha_{j}$. В случае $\alpha=0$ операторы циклической цепочки Дарбу являются конечнозонными. При $\alpha \neq 0$ уравнения на $f_{j}$ сводятся к уравнениям Пенлеве и их высшим аналогам, а соотношения (1) обязывают операторы $L_{j}$ иметь дискретный спектр, образованньй $r$ арифометическими прогрессиями (см. [1]).

Рассмотрим следующий дискретньй " $q$-аналог” цепочки Дарбу:

$$
L_{j}=A_{j} A_{j}^{+}-\alpha_{j}=q A_{j-1}^{+} A_{j-1}
$$

где $A_{j}=a_{j}+b_{j} T$ - разностные операторы на одномерной решетке $\mathbb{Z}, T$ - оператор сдвига: $\left(A_{j} f\right)(n)=a_{j}(n) f(n)+b_{j}(n) f(n+1), a_{j}(n), b_{j}(n) \in \mathbb{R} \backslash\{0\}$. Без ограничения общности можно считать, что $b_{j}(n)>0$ для всех $j, n$. Будем говорить, что цепочка (2) является циклической с периодом $r$ и сдвигом $s$, если для всех $j \geqslant 1$ выполнено

$$
L_{j+r}=T^{-s} L_{j} T^{s} .
$$

Ранее в литературе рассматривался в основном случай $s=0$. Однако в работе [2], а также в [3] указывалась возможность “обратной” факторизации, т.е. замены операторов $A_{j}=a_{j}+b_{j} T$ на операторы вида $A_{j}=a_{j} T^{+}+b_{j}$, что фактически эквивалентно рассмотрению "прямой” факторизации при $s=r$ (оператор $L_{j}$ заменяется на $T^{-j} L_{j} T^{j}$ ). Общая постановка с произвольньм $s$, по-видимому, не рассматривалась. В литературе изучались следующие случаи.

1. $\alpha=0, q=1, r$ - произвольное [2]. Операторы $L_{j}$ являются конечнозонными.

2. $\alpha>0, q=1, r=1$ (разностньй аналог гармонического осциллятора) [2]. В этом случае симметричных операторов $L_{j}$ в пространстве функций на всей решетке $\mathbb{Z}$ не существует, однако имеются решения на "полупрямой” $\mathbb{Z}>0$. Спектр оператора $L+\frac{\alpha}{2}$ такой же, как спектр обычного гармонического осциллятора, $\lambda_{k}=\alpha\left(k+\frac{1}{2}\right)$, собственные функции выражаются через многочлены Шарлье и потому образуют полное семейство в $\mathscr{L}_{2}\left(\mathbb{Z}_{>0}\right)$.

3. $r=1, \alpha>0,0<q<1$ (или $\alpha<0,1<q$ ) [2], [4] (q-осциллятор). Оператор $L$ имеет дискретный спектр в промежутке $\left[0, \frac{q \alpha}{1-q}\right)$ (или $\left(0, \frac{q \alpha}{q-1}\right)$ в случае $\left.q>1\right)$, образующий " $q$-арифметическую прогрессию". Как отмечается в [2], оператор $L$ в этом случае неограничен и, по-видимому, имеет непрерывньй спектр в области $\left(\frac{q \alpha}{1-q}, \infty\right)$.

4. В работе [5] рассмотрена версия $q$-осциллятора в виде разностного оператора на всей прямой, которая в нашей постановке может быть интерпретирована как случай $s=1, r=2, \alpha_{1}=\alpha_{2}$ ( $\alpha$ и $q$ такие же, как в предыдущем случае). В [5] указано частное решение, обладающее дополнительной симметрией относительно начала координат. Особенность данного случая в том, что оператор $L$ является ограниченным и не имеет непрерьвного спектра.

Здесь мы укажем, что последнее свойство наблюдается в случае цепочки произвольной четной длины при $s=r / 2$, и приведем явньй вид общего решения задачи в случае $s=1, r=2$.

Теорема. Для произвольных четного $r$, положительных $\alpha_{1}, \ldots, \alpha_{r}, 0<q<1$ задача (2), (3) при $s=r / 2$ имеет $r$-параметрическое семейство решений. При этом для всех $j$ оператор $L_{j}$ ограничен и имеет только дискретный спектр $\left\{\lambda_{j, 0}, \lambda_{j, 1}, \ldots\right\}$ в проме-

Работа выполнена при частичной поддержке Российского фонда фундаментальных исследований (грант № 99-01-00090). 
жутке $[0,\|L\|)$, вычисляемьй по схеме Дарбу: $\lambda_{j, 0}=0, \quad \lambda_{j+1, k+1}=q\left(\lambda_{j, k}+\alpha_{j}\right)$, $\lambda_{j+r, k}=\lambda_{j, k}$. Для каждого $j$ собственные функции операторов $L_{j}$, также вычисляемье по схеме Дарбу: $A_{j-1} \psi_{j, 0}=0, \psi_{j+1, k+1}=A_{j}^{+} \psi_{j, k}$, образуют полное семейство в $\mathscr{L}_{2}(\mathbb{Z})$.

Аналогичное утверждение имеет место при $\alpha_{1}, \ldots, \alpha_{r}<0,1<q$ (в этом случае нуль не входит в спектр операторов $L_{j}$ ).

ПреДЛОЖЕнИЕ. При $r=2=2 s, \alpha_{1}, \alpha_{2}>0,0<q<1$ общее решение задачи (2), (3) имеет вид: $a_{1}(n)=\epsilon \sqrt{\xi_{2 n}}, b_{1}(n)=\sqrt{\eta_{2 n+1}}, a_{2}(n)=\epsilon \sqrt{\xi_{2 n-1}}, b_{2}(n)=\sqrt{\eta_{2 n}}$, əде $\epsilon= \pm 1$,

$$
\begin{gathered}
\xi_{n}=\frac{1}{2} \frac{c_{n}-2 \varkappa q^{-n-\varphi-\frac{1}{2}}+c_{n+1} q^{-2 n-2 \varphi-1}}{\left(1-q^{-2(n+\varphi)}\right)\left(1-q^{-2(n+\varphi+1)}\right)}, \eta_{n}=\frac{1}{2} \frac{c_{n+1}-2 \varkappa q^{n+\varphi+\frac{1}{2}}+c_{n} q^{2 n+2 \varphi+1}}{\left(1-q^{2(n+\varphi)}\right)\left(1-q^{2(n+\varphi+1)}\right)} \\
c_{n}=\frac{\alpha_{1}+\alpha_{2}}{1-q}+(-1)^{n} \frac{\alpha_{1}-\alpha_{2}}{1+q}
\end{gathered}
$$

$\varphi$ - произвольный параметр, а параметр ж удовлетворяет ограничениям

$c_{[\varphi]} q^{-\theta}+c_{[\varphi]-1} q^{\theta}<2 \varkappa<\min \left(c_{[\varphi]} q^{\theta+1}+c_{[\varphi]-1} q^{-\theta-1}, c_{[\varphi]} q^{\theta-1}+c_{[\varphi]-1} q^{-\theta+1}\right)$ npu $\varphi \notin \mathbb{Z}$ u $2 \varkappa=c_{\varphi} q^{\frac{1}{2}}+c_{\varphi-1} q^{-\frac{1}{2}}$, если $\varphi \in \mathbb{Z}$ (в этом случае дроби (4) следует сократить на $1-q^{2(n+\varphi)} n p u n \equiv \varphi(\bmod 2)$ ина $\left.1-q^{2(n+\varphi+1)} n p u n \equiv \varphi+1(\bmod 2)\right)$, əде $\theta=\varphi-[\varphi]-\frac{1}{2}$, $[\varphi]$ обозначает челую часть числа $\varphi$.

НАБлЮдЕнИЕ. В случае $r=2, \alpha_{1}=\alpha_{2}$ операторы $L_{j}+\frac{\alpha_{j}}{2}$, построенные по указанным решениям, сходятся при $q \rightarrow 1$ к оператору гармонического осциллятора в следующем смысле. Положим $\varphi=0, \epsilon=-1, q=\exp \left(-\frac{\alpha_{1}}{4} h^{2}\right), x=n h, T=\exp \left(h \frac{d}{d x}\right)$ и будем считать $n$ вещественным в формулах $(4)$, а оператор $L_{j}$ - разностным оператором на всей прямой $\mathbb{R}$. Тогда для $f \in C^{2}(\mathbb{R})$ будем иметь

$$
\left(L_{1,2}+\frac{\alpha_{1}}{2}\right) f(x)=\left(-\frac{d^{2}}{d x^{2}}+\frac{\alpha_{1}^{2}}{4} x^{2}\right) f(x)+o(h) .
$$

Аналогично, в случае $\alpha_{1} \neq \alpha_{2}$ оператор $L_{j}$ сходится к

$$
-\frac{d^{2}}{d x^{2}}+\frac{\left(\alpha_{j}+\alpha_{j+1}\right)^{2}}{16} x^{2}-\frac{\alpha_{j}}{2}-\frac{\left(\alpha_{j}-\alpha_{j+1}\right)\left(\alpha_{j}+3 \alpha_{j+1}\right)}{4\left(\alpha_{j}+\alpha_{j+1}\right)^{2} x^{2}},
$$

где $\alpha_{j+2}=\alpha_{j}$.

В случаях 2, 3, упомянутых выше, такая связь между дискретной и непрерьвной задачами не имеет места. Таким образом, рассматриваемый нами случай $s=r / 2$ дает в каком-то смысле "правильную" дискретизацию обычной цепочки Дарбу.

Мы надеемся доказать, что такого рода сходимость $q$-цепочки к обычной цепочке Дарбу имеет место и в случае произволного четного $r$. Численньй эксперимент подтверждает сходимость $q$-цепочки периода 6 к обычной цепочке Дарбу периода 3 в случае $\alpha_{j}=\alpha_{j+3}$.

\section{СПИСОК ЛИТЕРАТУРЫ}

[1] А. П. Веселов, А. Б. Шабат // Функц. анализ и его прил. 1993. Т. 27. № 2. С. 1-21. [2] S.P. Novikov, I. A. Taimanov. Appendix II in S. P. Novikov, A. P. Veselov // Amer. Math. Soc. Transl. Ser. 2. 1997. V. 179. P. 109-132. [3] С. П. Новиков, И. А. Дынников // УМH. 1997. Т. 52. № 5. С. 175-234. [4] V. Spiridonov, L. Vinet, A. Zhedanov // Lett. Math. Phys. 1993. V. 29. № 1. P. 63-73. [5] N. Atakishiyev, A. Frank, K. Wolf // J. Math. Phys. 1994. V. 35. № 7. P. 3253-3260.

Московский государственньй

Принято редколлегией университет им. М.В. Ломоносова 23.09 .2002 